\title{
ZFOURGE: UV to FIR Luminosities and Dust Attenuation Determined From $\sim 4000$ K-selected Galaxies at $1<z<3$
}

\author{
Ben Forrest*, Kim-Vy H. Tran \\ George P. and Cynthia W. Mitchell Institute for Fundamental Physics and Astronomy, \\ Department of Physics and Astronomy, Texas A\&M University, College Station, TX 77843, USA \\ E-mail: bforrestephysics.tamu.edu
}

\section{The ZFOURGE Collaboration}

\section{The CANDELS-Herschel Team}

\begin{abstract}
We build a set of composite galaxy SEDs by de-redshifting and scaling multi-wavelength photometry from galaxies in the ZFOURGE survey, covering the CDFS, COSMOS, and UDS fields. From a sample of $4000 K_{s}$-band selected galaxies, we create 38 composite galaxy SEDs that yield continuous low-resolution spectra $(R \sim 45)$ over the range 0.1-4 $\mu \mathrm{m}$. Additionally, we include far infrared photometry from the Spitzer Space Telescope and the Herschel Space Observatory to characterize the infrared properties of our diverse set of composite SEDs. From these composite SEDs we analyze the rest-frame $U V J$ colors, as well as the ratio of IR to UV light (IRX) and the UV slope $(\beta)$ in the IRX $-\beta$ dust relation at $1<z<3$. While blue star-forming composite SEDs show IRX and $\beta$ values consistent with local relations, dusty star-forming composite SEDs are offset above the local IRX $-\beta$ relations by as much as 0.5 dex, i.e., dusty star-forming galaxies have more UV flux (are bluer). We measure a tight linear relation between rest-frame $U V J$ colors and dust attenuation for star-forming composites, providing a direct method for estimating dust content from either $(U-V)$ or $(V-J)$ rest-frame colors for galaxies at intermediate redshifts.
\end{abstract}

BASH 2015

18 - 20 October, 2015

The University of Texas at Austin, USA

\footnotetext{
* Speaker.

${ }^{\dagger}$ http://zfourge.tamu.edu/

† This work includes data gathered with the 6.5 meter Magellan Telescopes located at Las Campanas Observatory, Chile.
} 


\section{Method}

Galaxies are grouped together by scaling and de-redshifting public photometry from the CDFS, COSMOS, and UDS fields covered by the ZFOURGE survey [1]. We then compute synthetic photometric points using EAZY [2] and compare these to find similar galaxies, termed analogs [3, 4]. We take median points of de-redshifted, scaled analog photometry to create composite SEDs with $R \sim 45$ in the optical (Figure 1). Rest-frame colors are calculated using EAZY to place composites on the $U V J$ diagram (Figure 2). We compute IR and UV fluxes to derive an IRX- $\beta$ relation for our $1<z<3$ sample (Figure 3 ).

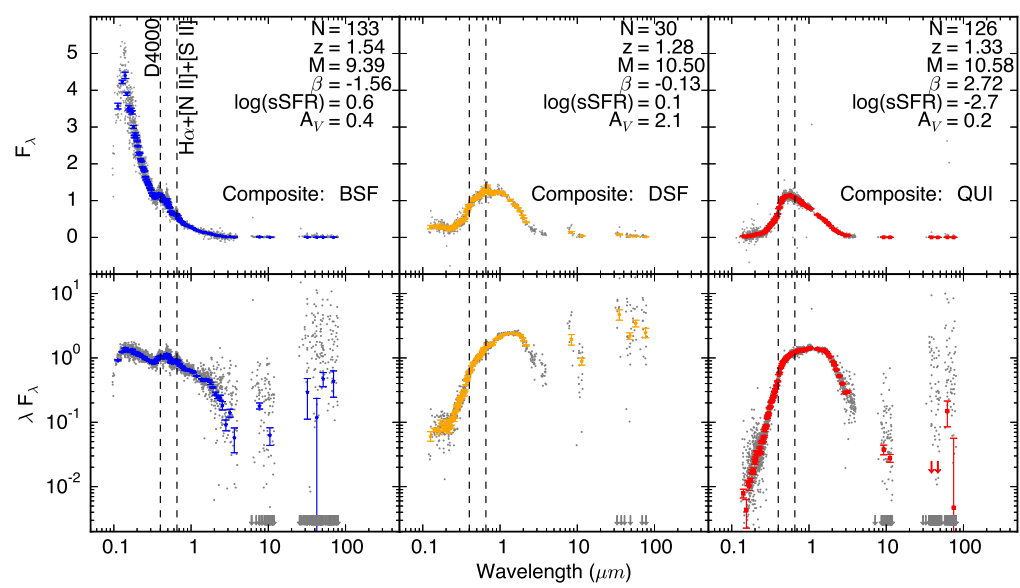

Figure 1: Examples of our composite SEDs, including a blue star-forming composite SED (BSF), a dusty star-forming composite SED (DSF), and a quiescent composite SED (QUI). Colored points represent the composite SEDs with NMAD scatter on the median as errorbars, while the gray points are the de-redshifted, scaled photometry from observations.

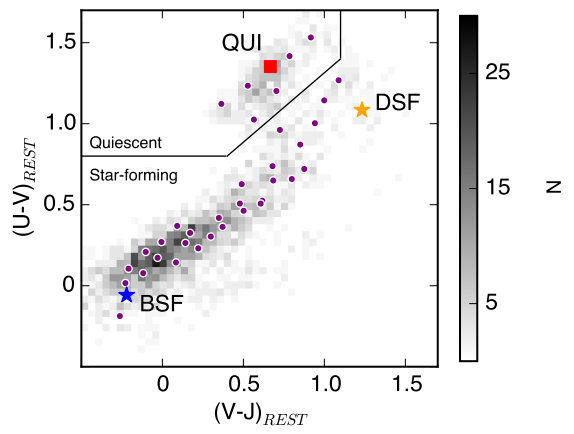

Figure 2: $U V J$ diagram of our composites, also showing locations of analog galaxies as a 2D histogram. Our composites track the underlying population nicely, including blue and red star forming galaxies (lower left and upper right) and quiescent galaxies (upper left).

\section{Analysis}

We find a discrepancy between our IRX $-\beta$ fit and fits from the literature calculated for local samples [5, 6]. This relation parameterizes the efficiency with which UV light from young, massive 


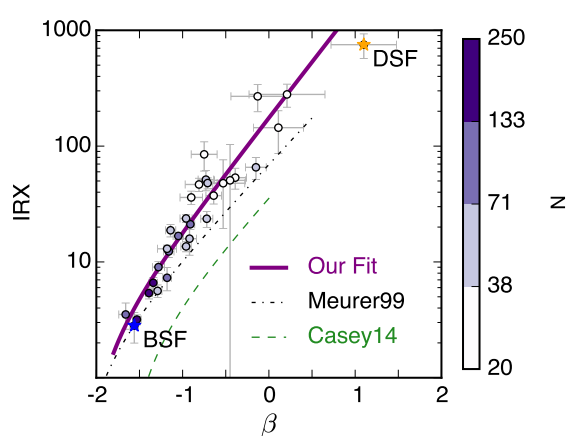

Figure 3: IRX $-\beta$ diagram, showing our fit (purple) compared to fits to the local samples of Meurer, et al. (1999; black) and Casey, et al. (2014; green). Our fit diverges from these relations, particularly for dusty star-forming galaxies.
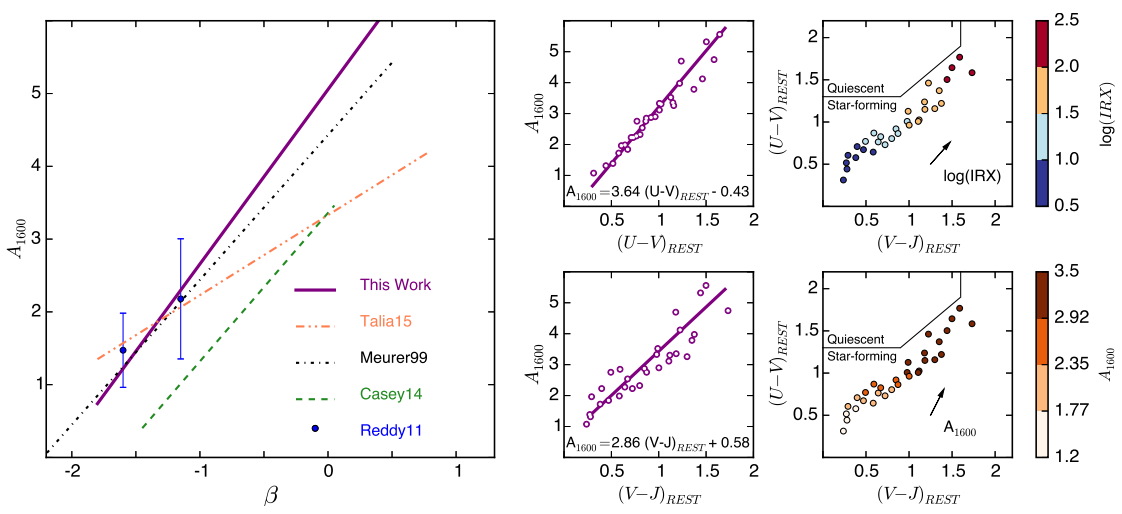

Figure 4: Dust attenuation as a function of UV slope (left). Relation between UV dust attenuation derived from IRX $-\beta$ and $U V J$ rest-frame colors (center). $U V J$ diagrams colored by IRX and $A_{1600}$ (right).

stars is transformed into IR radiation by dust. The discrepancy suggests that dusty star-forming galaxies at intermediate redshifts are obscured differently than local galaxies, and are intrinsically bluer.

We also find trends with low scatter between dust attenuation in the UV and rest-frame UVJ colors for star-forming galaxies, which can be used to parameterize dust of star-forming galaxies without spectra. Future work will explore other properties of our composite SEDs, such as $\mathrm{H} \alpha$ equivalent widths and morphological characteristics.

\section{References}

[1] Straatman, C. M. S., Spitler, L. R., Quadri, R. F., et al. 2016, submitted

[2] Brammer, G. B., van Dokkum, P. G., \& Coppi, P. 2008, The Astrophysical Journal, 686, 1503

[3] Forrest, B., Tran, K.-V. H., Tomczak, A. R., Broussard, A., et al. submitted

[4] Kriek, M., van Dokkum, P. G., Whitaker, K. E., et al. 2011, The Astrophysical Journal, 743, 168

[5] Meurer, G. R., Heckman, T. M., \& Calzetti, D. 1999, The Astrophysical Journal, 521, 64

[6] Casey, C. M., Scoville, N. Z., Sanders, D. B., et al. 2014, The Astrophysical Journal, 796, 95 